\title{
Unrelated Question Model in Sensitive Multi-Character Surveys
}

\author{
Sukhjinder Singh Sidhu ${ }^{a}$, Mohan Lal Bansal ${ }^{a}$, Jong-Min Kim ${ }^{1, b}$, Sarjinder Singh ${ }^{c}$ \\ aDept.of Mathematics, Statistics and Physics, Punjab Agricultural Univ., \\ ${ }^{b}$ Div. of Science and Mathematics, Univ. of Minnesota, ${ }^{C}$ Dept. of Mathematics, Texas A\&M Univ.
}

\begin{abstract}
The simplicity and wide application of Greenberg et al. (1971) prompts to propose a set of alternative estimators of population total for multi-character surveys that elicit simultaneous information on many sensitive study variables. The proposed estimators take into account the already known rough value of the correlation coefficient between $Y$ (the characteristic under study) and $p$ (the measure of size). These estimators are biased, but it is expected that the extent of bias will be smaller, since the proposed estimators are suitable for situations in between those optimum for the usual estimators and the estimators based on multi-characters for no correlation. The relative efficiency of the proposed estimators has been studied under a super population model through empirical study. It has been found through simulation study that a choice of an unrelated variable in the Greenberg et al. (1971) model could be made based on its correlation with the auxiliary variable used at estimation stage in multi-character surveys.
\end{abstract}

Keywords: Total estimation, RRT, sensitive multi-characteristics, mean square error, super population model, cost aspects and empirical study.

\section{Introduction}

The well-known Hansen and Hurwitz (1943) estimator of population total for probability proportional to size and with replacement sampling (PPSWR) is given by

$$
\widehat{Y}_{\mathrm{HH}}=\frac{1}{n} \sum_{i=1}^{n} \frac{y_{i}}{p_{i}}, \quad \text { where } p_{i}=x_{i}\left(\sum_{i=1}^{N} x_{i}\right)^{-1} \text {. }
$$

In sample surveys of many variables, some of the study variables may be poorly correlated with the selection probabilities. In this the use of usual estimators available in literature results in larger variance. Rao (1966) has provided alternative estimators when the study variable and size measure are unrelated and demonstrated that these alternative estimators are more efficient though biased. But Rao's (1966) model is not commonly encountered in practice since the correlation is not always zero. Bansal and Singh (1985) developed a transformed estimator of population total suitable for the characteristics covering entire range of positive correlation. Amahia et al. (1989) suggested simple alternatives to the transformations in Bansal and Singh (1985). The transformations of selection probabilities used are as follows: 


$$
\begin{array}{lll}
p_{i 0}^{*}=\frac{1}{N}, & & {[\text { Rao (1966)] }} \\
p_{i 1}^{*}=\left(1+\frac{1}{N}\right)^{1-\rho}\left(1+p_{i}\right)^{\rho}-1, & & {[\text { Bansal and Singh (1985)] }} \\
p_{i 2}^{*}=\frac{1}{N}(1-\rho)+\rho p_{i}, & & {[\text { Amahia et al. (1989)] }} \\
p_{i 3}^{*}=\left(\frac{1}{N}\right)^{1-\rho} p_{i}^{\rho}, & & \text { [Amahia et al. (1989)] } \\
p_{i 4}^{*}=\left\{N(1-\rho)+\frac{\rho}{p_{i}}\right\}^{-1}, & & {[\text { Amahia et al. (1989)] }} \\
p_{i 5}^{*}=\frac{1}{N}\left(1-\rho^{\frac{1}{3}}\right)+\rho^{\frac{1}{3}} p_{i}, & & {[\text { Grewal et al. (1997)]. }}
\end{array}
$$

On the basis of these transformations, following types of estimators of population total $Y$ under PPSWR sampling are available in the literature:

$$
\left(\widehat{Y}_{\mathrm{pps}}\right)_{h}=\frac{1}{n} \sum_{i=1}^{n} \frac{y_{i}}{p_{i h}^{*}}, \quad h=1,2,3,4,5 .
$$

The transformations $p_{i h}^{*}(h=1,2,3,4,5)$ at $(1.3)$ to $(1.7)$ of the selection probabilities $p_{i}$ are useful for positive correlation between $y_{i}$ and $p_{i}$ variables, whereas transformation (1.2) is useful under no correlation situation. Interestingly for $\rho=0, p_{i h}^{*}(h=1,2,3,4,5)$ reduce to $p_{i 0}^{*}$ at $(1.2)$ and for $\rho=1$ these transformations reduce to original selection probabilities $p_{i}$. For detail one can refer to Arnab (2001) and Singh (2003).

The surveys on human population had established the fact that the direct question about sensitive characters often result in either refusal to respond or falsification of the answer. This can bias the estimates. Warner (1965) developed an interviewing procedure designed to reduce or eliminate this bias and called it as Randomized Response Technique(RRT). It is beneficial to combine multi-characteristics and RRT. Bansal et al. (1994) and Grewal et al.(1997) had discussed the multicharacteristics in RRT to estimate population total.

It was felt that the confidence of the respondents in anonymity provided by RRT and hence reliability of their responses, might be further enhanced if one of the two question belong to non sensitive, innocuous attribute unrelated to the sensitive characteristics. Greenberg et al. (1971) developed the work for quantitative responses and found that his unrelated question technique was more efficient than the Warner (1965) model.

\section{UQ Model}

In the quantitative unrelated question (UQ) random response model, using two questions, the overall distribution of responses is comprised of numerical answers to both questions, the answers being indistinguishable as to question. This distribution is a mixture of two pure distributions, which must be statistically separated to provide meaningful estimates of the parameters of interest. The population means of both the sensitive $(Y)$ and unrelated non-sensitive $(U)$ variables are $\mu_{y}$ and $\mu_{U}$ with their respective variances $\sigma_{y}^{2}$ and $\sigma_{U}^{2}$. 
When the value of $U$ (total of unrelated character) is known in advance we select one sample of size $n$. The respondent in the sample is provided with a randomization device, with probability $T$ and $(1-T)$, respectively, consisting of sensitive and non sensitive statements:

(i) About how much money in dollars did the head of household, earn last year?

(ii) About how much average money in dollars do you think the head of a household of your size earns in a year?

The respondent selects randomly one of the two statements, unobserved by the interviewer, and reports the answer. Let response from $i^{t h}$ individual in the sample for the characteristic under study be denoted by $r_{i}$.

$$
r_{i}=T \quad y_{i}+(1-T) \mu_{i}
$$

with

$$
V\left(r_{i}\right)=T(1-T)\left(y_{i}-\mu_{i}\right)^{2} .
$$

Keeping in view the importance of this model, we extend the method to multi-character surveys to propose estimators of population total. The behavior of the proposed estimators has been examined under the super population model given below.

\section{Super Population Model}

A general super population model for sensitive characteristic under study is:

$$
Y_{i}=\beta p_{i}+e_{i}, \quad i=1,2, \ldots, N,
$$

where $e_{i}$ 's are the error terms such that:

$$
\begin{aligned}
E_{m}\left(e_{i} \mid p_{i}\right) & =0 \\
E_{m}\left(e_{i} e_{j} \mid p_{i} p_{j}\right) & =0
\end{aligned}
$$

and

$$
E_{m}\left(e_{i}^{2} \mid p_{i}\right)=a p_{i}^{g}, \quad a>0, g \geq 0 .
$$

Here $E_{m}\left(e_{i}^{2} \mid p_{i}\right)$ is the residual variances of $Y$ for given $p_{i}$. The expected value of this residual variance in the super population model is given by:

$$
E_{m}\left(a p_{i}^{g}\right)=a E_{m}\left(p_{i}^{g}\right)
$$

and when the infinite super population is simulated by a finite large population of $N$ units having the same characteristics it will be reduced to:

$$
E\left(a p_{i}^{g}\right)=\frac{a}{N} \sum_{i=1}^{N} p_{i}^{g} .
$$

Also the expected value of residual variance is known to be given by $\sigma_{y}^{2}\left(1-\rho^{2}\right)$.

Thus we have:

$$
\frac{a}{N} \sum_{i=1}^{N} p_{i}^{g}=\sigma_{y}^{2}\left(1-\rho^{2}\right)
$$


or

$$
\sigma_{y}^{2}=\frac{\frac{a}{N} \sum_{i=1}^{N} p_{i}^{g}}{1-\rho^{2}} .
$$

The value of the regression coefficient is given by:

$$
\beta^{2}=\rho^{2} \frac{\sigma_{y}^{2}}{\sigma_{p}^{2}}=\frac{\rho^{2}}{1-\rho^{2}}\left(\frac{a}{N} \sum_{i=1}^{N} \frac{p_{i}^{g}}{\sigma_{p}^{2}}\right),
$$

where

$$
\sigma_{p}^{2}=\frac{1}{N}\left(\sum_{i=1}^{N} p_{i}^{2}-\frac{\left(\sum_{i=1}^{N} p_{i}\right)^{2}}{N}\right) .
$$

The super population model for unrelated non-sensitive question is:

$$
U_{i}=\beta^{*} p_{i}+e_{i}^{*}, \quad i=1,2, \ldots, N,
$$

where $e_{i}^{*}$ 's are the error terms satisfying all the conditions at (3.2), (3.3) and (3.4).

It is assumed for simplicity that means of $Y_{i}$ and $U_{i}$ are different but the residual variances of $U$ for $p=p_{i}$, i.e. $E\left(e_{i}^{* 2} \mid p_{i}\right)$ is same as of $Y$.

Similarly

$$
\beta^{* 2}=\rho^{* 2} \frac{\sigma_{U}^{2}}{\sigma_{p}^{2}}=\frac{\rho^{* 2}}{1-\rho^{* 2}}\left(\frac{a}{N} \sum_{i=1}^{N} \frac{p_{i}^{g}}{\sigma_{p}^{2}}\right) .
$$

We first obtain the estimator of population total for PPSWR.

\section{Estimator $\left(\widehat{Y}_{1}\right)$}

When the value of $U$ (total of unrelated character) is known in advance we select one sample of size $n$. The respondents in the sample are provided with a randomization device consisting of sensitive and non sensitive statements with probability $T$ and $(1-T)$ respectively. The respondent selects randomly one of the two statements, unobserved by the interviewer and reports the answer. Let response from $i^{t h}$ individual in the sample for the characteristic under study be denoted by $r_{i}$.

$$
r_{i}=T y_{i}+(1-T) \mu_{i}
$$

The estimator of population total $\left(\widehat{Y}_{1}\right)$ for PPSWR is obtained as

$$
\widehat{Y}_{1}=\frac{1}{T}\left\{\frac{1}{n} \sum_{i=1}^{n} \frac{r_{i}}{p_{i}}-(1-T) U\right\} .
$$

The variance of the randomized response of $i^{\text {th }}$ individual is

$$
V\left(r_{i}\right)=T(1-T)\left(y_{i}-\mu_{i}\right)^{2} .
$$


Using this result it can be shown easily that the estimator $\left(\widehat{Y}_{1}\right)$ is unbiased. The variance of the estimator $\left(\widehat{Y}_{1}\right)$ is given in the following theorem.

Theorem 1. The variance of the estimator $\left(\widehat{Y}_{1}\right)$ given in $(4.2)$ is given by

$$
V\left(\widehat{Y}_{1}\right)=\frac{1-T}{n T} \sum_{i=1}^{N} \frac{\left(Y_{i}-U_{i}\right)^{2}}{p_{i}}+\frac{1}{n T^{2}} \sum_{i=1}^{N} p_{i}\left\{T\left(\frac{Y_{i}}{p_{i}}-Y\right)+(1-T)\left(\frac{U_{i}}{p_{i}}-U\right)\right\}^{2}
$$

Proof: Please see the Appendix A.

We now extend the theory for the estimator obtained above to propose the estimators of population total in case of multi-character surveys.

\section{Proposed Estimator $\left(\widehat{Y}_{2}\right)$}

The proposed estimators of population total $\left(\widehat{Y}_{2}\right)_{h}$ for multi-characteristics are given by

$$
\left(\widehat{Y}_{2}\right)_{h}=\frac{1}{T}\left\{\frac{1}{n} \sum_{i=1}^{n} \frac{r_{i}}{p_{i h}^{*}}-(1-T) U\right\},
$$

where $p_{i h}^{*}$ are defined in (1.2) to (1.7). The proposed estimators are biased and the bias in the the estimators $\left(\widehat{Y}_{2}\right)_{h}$ is given by

$$
B\left(\widehat{Y}_{2}\right)_{h}=\sum_{i=1}^{N}\left(\frac{p_{i}}{p_{i h}^{*}}-1\right)\left\{Y_{i}+\frac{(1-T)}{T} U_{i}\right\} .
$$

One can easily see that the variance of the estimator $\left(\widehat{Y}_{2}\right)_{h}$ is given by

$$
\begin{aligned}
V\left(\widehat{Y}_{2}\right)_{h}= & \frac{1-T}{n T} \sum_{i=1}^{N} \frac{\left(Y_{i}-U_{i}\right)^{2} p_{i}}{p_{i h}^{* 2}} \\
& +\frac{1}{n T^{2}}\left[\sum_{i=1}^{N} \frac{\left(T Y_{i}+(1-T) U_{i}\right)^{2} p_{i}}{p_{i h}^{* 2}}-\left\{\sum_{i=1}^{N} \frac{\left(T Y_{i}+(1-T) U_{i}\right) p_{i}}{p_{i h}^{*}}\right\}^{2}\right] .
\end{aligned}
$$

Please see the full derivation of the equation (5.3) in the Appendix A.

To obtain the expected Mean Square Error (MSE) of proposed estimators $\left(\widehat{Y}_{2}\right)_{h}$ under super population model we have the following theorem.

Theorem 2. The expected value of MSE of $\left(\widehat{Y}_{2}\right)_{h}$ under the superpopulation model is

$$
E_{m}\left[\operatorname{MSE}\left(\widehat{Y}_{2}\right)_{h}\right]=\frac{1}{n} A_{1}+A_{2}
$$

where

$$
\begin{aligned}
A_{1}= & {\left[\left(\frac{1-T}{T}\right)\left(\left(\beta-\beta^{*}\right)^{2} \sum_{i=1}^{N} \frac{p_{i}^{3}}{p_{i h}^{* 2}}+2 a \sum_{i=1}^{N} \frac{p_{i}^{g+1}}{p_{i h}^{* 2}}\right)+\frac{1}{T^{2}}\left\{a\left(T^{2}+(1-T)^{2}\right)\left(\sum_{i=1}^{N} \frac{p_{i}^{g+1}}{p_{i h}^{* 2}}-\sum_{i=1}^{N} \frac{p_{i}^{g+2}}{p_{i h}^{* 2}}\right)\right.\right.} \\
& \left.\left.+\left(\beta T+(1-T) \beta^{*}\right)^{2}\left(\sum_{i=1}^{N} \frac{p_{i}^{3}}{p_{i h}^{* 2}}-\left(\sum_{i=1}^{N} \frac{p_{i}^{2}}{p_{i h}^{*}}\right)^{2}\right)\right\}\right]
\end{aligned}
$$


and

$$
A_{2}=\left\{\left(\sum_{i=1}^{N} \frac{p_{i}^{2}}{p_{i h}^{*}}-1\right)\left(\beta+\frac{1-T}{T} \beta^{*}\right)\right\}^{2}+a\left(1+\frac{(1-T)^{2}}{T^{2}}\right) \sum_{i=1}^{N}\left(\frac{p_{i}^{g+2}}{p_{i h}^{* 2}}+p_{i}^{g}-2 \frac{p_{i}^{g+1}}{p_{i h}^{*}}\right)
$$

Proof: Please see the Appendix A.

When the value of non-sensitive question is known in advance. We choose the strategy, which for a fixed cost can estimate $Y$ with maximum accuracy. For this we find the minimum expected mean square error for fixed cost under super population model. This we do in the following theorem.

Theorem 3. Under superpopulation model, for the fixed cost $C_{0}$ the minimum expected mean square error of estimator $\left(\widehat{Y}_{2}\right)_{h}$ is given by

$$
E_{m}\left[\operatorname{MSE}\left(\widehat{Y}_{2}\right)_{h}\right]=\frac{C_{1}}{C_{0}} A_{1}+A_{2},
$$

where $A_{1}$ and $A_{2}$ are defined in (5.5) and (5.6) and $C_{1}$ cost of processing per unit in the sample.

Proof: Please see the Appendix A.

\section{Empirical Study}

To investigate into the performance of the proposed estimators we resort to an empirical study under super population model given in Section 3. For this the relative efficiency under unrelated question model(RE_h $h$ of the proposed estimators $\left(\widehat{Y}_{2}\right)_{h}$ for $h=1,2,3,4,5$ with respect to $\left(\widehat{Y}_{2}\right)_{0}$ is given by

$$
\left(\operatorname{RE} \_h\right)=\frac{E_{m}\left[\operatorname{MSE}\left(\widehat{Y}_{2}\right)_{0}\right]}{E_{m}\left[\operatorname{MSE}\left(\widehat{Y}_{2}\right)_{h}\right]} \times 100
$$

where symbols have their usual meanings. The probability associated with the statements in the device is 0.7 and 0.3 , respectively. The choice of $T=0.7$ in the Greenberg et al. (1971) seems to be a reasonable choice, because a very high value of $T$ may effect the respondents' coopeartion while asking a question through the randomization device. The density functions for the auxiliary variable, which is assumed to have correlation of values $\rho$ in the range 0 to 1 with the study variables, are presented in Table 1.

For the sensitive character value of correlation coefficient between $X$ and $Y$ is $\rho=0.1,0.2,0.3,0.4$, $0.5,0.6,0.7,0.8,0.9$, but for unrelated question, correlation coefficient $\rho^{*}=0.15,0.65,0.95$ is used. Note that $\rho^{*}$ is the value of correlation coefficient between the unrelated character variable and the auxiliary variable or say the selection probabilities $p_{i}$. Thus, it could always be feasible to select an unrelated character variable which may have either low, moderate or high value of correlation coefficient with the selection probabilities $p$, thus we considered only three such values of $\rho^{*}$. As the nature of the sensitive variables $y_{i}$ s remains unpredictable, thus we decided to consider entire range of values of the correlation coefficient $\rho$ between 0 and 1. A PPSWR sample of size 20 is considered as drawn from a population consisting of 100 respondents. The computations are given in Appendix B. The results obtained from these computations are given below in Table 2 .

From this table it is clear that the proposed estimators fare better than the usual estimator for all the $p_{i h}^{*}(h=1,2,3,4,5)$ in majority of the cases. It is to pointed out that if the value of $\rho^{*}$ is low 
Table 1: Density functions for various probability distributions

\begin{tabular}{|c|c|c|c|}
\hline Sr. No. & Distribution & Density function & Range \\
\hline 1 & Right Triangular & $f(x)=2(1-x)$ & $0 \leq x \leq 1$ \\
\hline 2 & Exponential & $f(x)=e^{-x}$ & $x \geq 0$ \\
\hline 3 & Chi-Square at $v=6$ & $f(x)=\frac{1}{2 \frac{y}{2} \Gamma_{v}} e^{-\frac{x}{2} x^{v-2}}$ & $x \geq 0$ \\
\hline 4 & Gamma, $\alpha=2, \beta=1$ & $f(x)=\frac{1}{\beta^{\alpha} \Gamma_{\alpha}} x^{\alpha-1} e^{-{ }^{x}}$ & $x \geq 0$ \\
\hline 5 & Normal & $f(x)=\frac{1}{\sigma \sqrt{2 \pi}} e^{-\frac{1}{2}\left(\begin{array}{c}x-\mu \\
\sigma\end{array}\right)^{2}}$ & $-\infty<x<+\infty$ \\
\hline 6 & Log Normal & $f(x)=\frac{1}{x \sqrt{2 \pi}} e^{-\frac{1}{2}(\log (x))^{2}}$ & $x>0$ \\
\hline 7 & Beta, $\alpha=3, \beta=2$ & $f(x)=\frac{1}{B(\alpha, \beta)} x^{\alpha-1}(1-x)^{\beta-1}$ & $0 \leq x \leq 1$ \\
\hline
\end{tabular}

Table 2: Correlation range for which the proposed estimator $\left(\widehat{Y}_{2}\right)_{h}$ of population total of a sensitive character is more efficient than usual estimator for $p_{i h}^{*}$ given in (1.2) to (1.7).

\begin{tabular}{c|c|c|c|c|c|c|c|c|c}
\hline \hline Distribution & \multicolumn{3}{|c|}{$g=0$} & \multicolumn{3}{c|}{$g=1$} & \multicolumn{3}{c}{$g=2$} \\
\hline$\rho^{*}$ & 0.15 & 0.65 & 0.95 & 0.15 & 0.65 & 0.95 & 0.15 & 0.65 & 0.95 \\
\hline Right Triangular & $0.2-0.9$ & $0.1-0.9$ & $0.1-0.9$ & $0.2-0.9$ & $0.1-0.9$ & $0.1-0.9$ & $0.2-0.9$ & $0.1-0.9$ & $0.1-0.9$ \\
\hline Exponential & $0.2-0.9$ & $0.1-0.9$ & $0.1-0.9$ & $0.2-0.9$ & $0.1-0.9$ & $0.1-0.9$ & $0.1-0.9$ & $0.1-0.9$ & $0.1-0.9$ \\
\hline Normal & $0.2-0.9$ & $0.2-0.9$ & $0.2-0.9$ & $0.2-0.9$ & $0.2-0.9$ & $0.2-0.9$ & $0.2-0.9$ & $0.2-0.9$ & $0.2-0.9$ \\
\hline Chi-sq, $v=6$ & $0.6-0.9$ & $0.5-0.9$ & $0.1-0.9$ & $0.5-0.9$ & $0.5-0.9$ & $0.1-0.9$ & $0.5-0.9$ & $0.4-0.9$ & $0.1-0.9$ \\
\hline Gamma (2,1) & $0.2-0.9$ & $0.1-0.9$ & $0.1-0.9$ & $0.2-0.9$ & $0.1-0.9$ & $0.1-0.9$ & $0.1-0.9$ & $0.1-0.9$ & $0.1-0.9$ \\
\hline Log Normal & $0.2-0.9$ & $0.1-0.9$ & $0.1-0.9$ & $0.1-0.9$ & $0.1-0.9$ & $0.1-0.9$ & $0.1-0.9$ & $0.1-0.9$ & $0.1-0.9$ \\
\hline Beta (3,2) & $0.5-0.9$ & $0.3-0.9$ & $0.1-0.9$ & $0.4-0.9$ & $0.3-0.9$ & $0.1-0.9$ & $0.4-0.9$ & $0.3-0.9$ & $0.1-0.9$ \\
\hline
\end{tabular}

or mderate, then a high value of correlation coefficient $\rho$ is required for the proposed estimators to efiicient in case of Chi-Square $(v=6)$ and Beta $(3,2)$ distribution. If the value of $\rho^{*}$ is high then the proposed estimator remains always more efficient. Interestingly, the choice of unrelated variable in the randomization device could be decided based on its correlation with the auxiliary variable used in the selection stage. Table 2 indicates that the value $\rho$ could be any value in the range $[0.1,0.9]$, the the proposed estimators performs better for $\rho^{*}=0.95$ in case of all the seven distributions considered in the simulation study.

\section{Acknowledgements}

The authors are thankful to the Editor, Associate Editor and two referees for their comments on the original version of the manuscript.

\section{Appendix A:}

Proof: (Proof of Theorem 1.) Let $E_{1}$ and $E_{2}$ denote the expected values with respect to sampling design and over randomization device respectively and let $V_{1}$ and $V_{2}$ be the corresponding variances, then

$$
\begin{aligned}
V\left(\widehat{Y}_{1}\right) & =E_{1} V_{2}\left(\widehat{Y}_{1}\right)+V_{1} E_{2}\left(\widehat{Y}_{1}\right) \\
& =E_{1} V_{2}\left[\frac{1}{T}\left\{\frac{1}{n} \sum_{i=1}^{n} \frac{r_{i}}{p_{i}}-(1-T) U\right\}\right]+V_{1} E_{2}\left[\frac{1}{T}\left\{\frac{1}{n} \sum_{i=1}^{n} \frac{r_{i}}{p_{i}}-(1-T) U\right\}\right]
\end{aligned}
$$


On using (4.3) and substituting $E\left(r_{i}\right)=y_{i} T+(1-T) u_{i}$, we have

$$
\begin{aligned}
V\left(\widehat{Y}_{1}\right)= & E_{1}\left[\frac{1}{T^{2}}\left\{\frac{1}{n^{2}} \sum_{i=1}^{n} \frac{T(1-T)\left(y_{i}-u_{i}\right)^{2}}{p_{i}^{2}}\right\}\right] \\
& +V_{1}\left[\frac{1}{T}\left\{\frac{1}{n} \sum_{i=1}^{n} \frac{y_{i} T+(1-T) u_{i}}{p_{i}}-(1-T) U\right\}\right] \\
= & \frac{1-T}{n T} \sum_{i=1}^{N} \frac{\left(Y_{i}-U_{i}\right)^{2}}{p_{i}}+\frac{1}{n T^{2}} \sum_{i=1}^{N} p_{i}\left\{T\left(\frac{Y_{i}}{p_{i}}-Y\right)+(1-T)\left(\frac{U_{i}}{p_{i}}-U\right)\right\}^{2} .
\end{aligned}
$$

\section{Full derivation of the equation 5.3:}

Let $E_{1}$ and $E_{2}$ denote the expected values with respect to sampling design and over randomization device respectively and let $V_{1}$ and $V_{2}$ be the corresponding variances, then

$$
\begin{aligned}
V\left(\widehat{Y}_{2}\right)_{h} & =E_{1} V_{2}\left(\widehat{Y}_{2}\right)+V_{1} E_{2}\left(\widehat{Y}_{2}\right) \\
& =E_{1} V_{2}\left[\frac{1}{T}\left\{\sum_{i=1}^{n} \frac{r_{i}}{p_{i}^{*}}-(1-T) U\right\}\right]+V_{1} E_{2}\left[\frac{1}{T}\left\{\frac{1}{n} \sum_{i=1}^{n} \frac{r_{i}}{p_{i}^{*}}-(1-T) U\right\}\right] \\
& =E_{1}\left[\frac{1}{T^{2}}\left\{\frac{1}{n^{2}} \sum_{i=1}^{n} \frac{V_{2}\left(r_{i}\right)}{p_{h i}^{* 2}}\right\}\right]+V_{1}\left[\frac{1}{T}\left\{\frac{1}{n} \sum_{i=1}^{n} \frac{E_{2}\left(r_{i}\right)}{p_{i h}^{*}}-(1-T) U\right\}\right] .
\end{aligned}
$$

On using (4.3) and substituting $E\left(r_{i}\right)=y_{i} T+(1-T) u_{i}$, we have

$$
\begin{aligned}
V\left(\widehat{Y}_{2}\right)_{h}= & E_{1}\left[\frac{1}{T^{2}}\left\{\frac{1}{n^{2}} \sum_{i=1}^{n} \frac{T(1-T)\left(y_{i}-u_{i}\right)^{2}}{p_{i h}^{* 2}}\right\}\right] \\
& +V_{1}\left[\frac{1}{T}\left\{\frac{1}{n} \sum_{i=1}^{n} \frac{y_{i} T+(1-T) u_{i}}{p_{i h}^{*}}-(1-T) U\right\}\right] \\
= & E_{1}\left[\frac{1}{T^{2}}\left\{\frac{1}{n^{2}} \sum_{i=1}^{n} \frac{T(1-T)\left(y_{i}-u_{i}\right)^{2}}{p_{i h}^{* 2}}\right\}\right]+\frac{1}{n^{2} T^{2}} V_{1}\left\{\sum_{i=1}^{n} \frac{y_{i} T+(1-T) u_{i}}{p_{i h}^{*}}\right\} \\
= & \frac{1-T}{n T} \sum_{i=1}^{N} \frac{\left(Y_{i}-U_{i}\right)^{2} p_{i}}{p_{i h}^{* 2}}+\frac{1}{n T^{2}} \sum_{i=1}^{N} p_{i}\left\{\frac{T Y_{i}+(1-T) U_{i}}{p_{i h}^{*}}-\sum_{i=1}^{N} \frac{T Y_{i}+(1-T) U_{i}}{p_{i h}^{*}} p_{i}\right\}^{2} \\
= & \frac{1-T}{n T} \sum_{i=1}^{N} \frac{\left(Y_{i}-U_{i}\right)^{2} p_{i}}{p_{i h}^{* 2}} \\
& +\frac{1}{n T^{2}}\left[\sum_{i=1}^{N} \frac{\left(T Y_{i}+(1-T) U_{i}\right)^{2} p_{i}}{p_{i h}^{* 2}}-\left\{\sum_{i=1}^{N} \frac{\left(T Y_{i}+(1-T) U_{i}\right) p_{i}}{p_{i h}^{*}}\right\}^{2}\right],
\end{aligned}
$$

which proves the equation.

Proof: (Proof of Theorem 2.) We know that

$$
E_{m}\left\{\operatorname{MSE}\left(\widehat{Y}_{2}\right)_{h}\right\}=E_{m}\left\{\mathrm{~V}\left(\widehat{Y}_{2}\right)\right\}+E_{m}\left\{\mathrm{~B}\left(\widehat{Y}_{2}\right)_{h}\right\}^{2}
$$


Thus we have

$$
E_{m}\left\{\operatorname{MSE}\left(\widehat{Y}_{2}\right)_{h}\right\}=\frac{(1-T) E_{m}\left(B_{1}\right)}{n T}+\frac{E_{m}\left(B_{2}\right)-E_{m}\left(B_{3}\right)}{n T^{2}}+E_{m}\left\{B\left(\widehat{Y}_{2}\right)_{h}\right\}^{2},
$$

where

$$
B_{1}=\sum_{i=1}^{N} \frac{\left(Y_{i}-U_{i}\right)^{2}}{p_{i h}^{* 2}} p_{i}, \quad B_{2}=\sum_{i=1}^{N} \frac{\left\{T Y_{i}+(1-T) U_{i}\right\}^{2}}{p_{i h}^{* 2}} p_{i}
$$

and

$$
B_{3}=\left\{\sum_{i=1}^{N} \frac{T Y_{i}+(1-T) U_{i}}{p_{i h}^{*}} p_{i}\right\}^{2}
$$

Under the superpopulation model, we have

$$
B_{1}=\sum_{i=1}^{N} \frac{\left(\beta p_{i}+e_{i}-\beta^{*} p_{i}-e_{i}^{*}\right)^{2}}{p_{i h}^{* 2}} p_{i}=\sum_{i=1}^{N} \frac{\left(\beta-\beta^{*}\right)^{2} p_{i}^{2}+\left(e_{i}-e_{i}^{*}\right)^{2}+2\left(\beta-\beta^{*}\right) p_{i}\left(e_{i}-e_{i}^{*}\right)}{p_{i h}^{* 2}} p_{i} .
$$

Thus

$$
E_{m}\left(B_{1}\right)=\left(\beta-\beta^{*}\right)^{2} \sum_{i=1}^{N} \frac{p_{i}^{3}}{p_{i h}^{* 2}}+2 a \sum_{i=1}^{N} \frac{p_{i}^{g+1}}{p_{i h}^{* 2}}
$$

Now

$$
\begin{aligned}
B_{2}= & \left\{\sum_{i=1}^{N} \frac{T^{2} Y_{i}^{2}+(1-T)^{2} U_{i}^{2}+2 T(1-T) Y_{i} U_{i}}{p_{i h}^{* 2}} p_{i}\right\} \\
= & \sum_{i=1}^{N} \frac{T^{2}\left(\beta p_{i}+e_{i}\right)^{2}+(1-T)^{2}\left(\beta^{*} p_{i}+e_{i}^{*}\right)^{2}+2 T(1-T)\left(\beta p_{i}+e_{i}\right)\left(\beta^{*} p_{i}+e_{i}^{*}\right)}{p_{i h}^{* 2}} p_{i} \\
= & \sum_{i=1}^{N} \frac{T^{2}\left(\beta^{2} p_{i}^{2}+e_{i}^{2}+2 \beta p_{i} e_{i}\right)+(1-T)^{2}\left(\beta^{* 2} p_{i}^{2}+e_{i}^{* 2}+2 \beta^{*} p_{i} e_{i}^{*}\right)}{p_{i h}^{* 2}} p_{i} \\
& +2 \sum_{i=1}^{N} \frac{T(1-T)\left(\beta \beta^{*} p_{i}^{2}+\beta p_{i} e_{i}^{*}+\beta^{*} p_{i} e_{i}+e_{i} e_{i}^{*}\right)}{p_{i h}^{* 2}} p_{i} .
\end{aligned}
$$

Thus, we have

$$
E_{m}\left(B_{2}\right)=\left\{T^{2}+(1-T)^{2}\right\} a \sum_{i=1}^{N} \frac{p_{i}^{g+1}}{p_{i h}^{* 2}}+\left\{\beta T+(1-T) \beta^{*}\right\}^{2} \sum_{i=1}^{N} \frac{p_{i}^{3}}{p_{i h}^{* 2}} .
$$

Now

$$
B_{3}=\left\{\sum_{i=1}^{N} \frac{T Y_{i}+(1-T) U_{i}}{p_{i h}^{*}} p_{i}\right\}^{2}=\left\{T \sum_{i=1}^{N} \frac{Y_{i} p_{i}}{p_{i h}^{*}}+(1-T) \sum_{i=1}^{N} \frac{U_{i} p_{i}}{p_{i h}^{*}}\right\}^{2}
$$


Using the superpopulation models, we have Using the superpopulation models, we have

$$
\begin{aligned}
B_{3}= & T^{2}\left\{\sum_{i=1}^{N} \frac{\left(\beta p_{i}+e_{i}\right) p_{i}}{p_{i h}^{*}}\right\}^{2}+(1-T)^{2}\left\{\sum_{i=1}^{N} \frac{\left(\beta^{*} p_{i}+e_{i}^{*}\right) p_{i}}{p_{i h}^{*}}\right\}^{2} \\
& +2 T(1-T)\left\{\sum_{i=1}^{N} \frac{\left(\beta^{*} p_{i}+e_{i}^{*}\right) p_{i}}{p_{i h}^{*}}\right\}\left\{\sum_{i=1}^{N} \frac{\left(\beta p_{i}+e_{i}\right) p_{i}}{p_{i h}^{*}}\right\} \\
= & T^{2}\left\{\beta \sum_{i=1}^{N} \frac{p_{i}^{2}}{p_{i h}^{*}}+\sum_{i=1}^{N} \frac{e_{i} p_{i}}{p_{i h}^{*}}\right)^{2}+(1-T)^{2}\left(\beta^{*} \sum_{i=1}^{N} \frac{p_{i}^{2}}{p_{i h}^{*}}+\sum_{i=1}^{N} \frac{e_{i}^{*} p_{i}}{p_{i h}^{*}}\right)^{2} \\
& +2 T(1-T)\left(\beta \sum_{i=1}^{N} \frac{p_{i}^{2}}{p_{i h}^{*}}+\sum_{i=1}^{N} \frac{e_{i} p_{i}}{p_{i h}^{*}}\right)\left(\beta^{*} \sum_{i=1}^{N} \frac{p_{i}^{2}}{p_{i h}^{*}}+\sum_{i=1}^{N} \frac{e_{i}^{*} p_{i}}{p_{i h}^{*}}\right)^{N} \\
= & T^{2}\left\{\beta^{2}\left(\sum_{i=1}^{N} \frac{p_{i}^{2}}{p_{i h}^{*}}\right)^{2}+\sum_{i=1}^{N} \frac{e_{i}^{2} p_{i}^{2}}{p_{i h}^{* 2}}+\sum_{j \neq i=1}^{N} \frac{p_{i} p_{j} e_{i} e_{j}}{p_{i h}^{*} p_{j h}^{*}}+2 \beta\left(\sum_{i=1}^{N} \frac{p_{i}^{2}}{p_{i h}^{*}} \sum_{i=1}^{N} \frac{p_{i} e_{i}}{p_{i h}^{*}}\right)\right\} \\
& +(1-T)^{2}\left\{\beta^{* 2}\left(\sum_{i=1}^{N} \frac{p_{i}^{2}}{p_{i h}^{*}}\right)^{2}+\sum_{i=1}^{N} \frac{e_{i}^{* 2} p_{i}^{2}}{p_{i h}^{* 2}}+\sum_{j \neq i=1}^{N} \frac{p_{i} p_{j} e_{i}^{*} e_{j}^{*}}{p_{i h}^{*} p_{j h}^{*}}+2 \beta^{*}\left(\sum_{i=1}^{N} \frac{p_{i}^{2}}{p_{i h}^{*}} \sum_{i=1}^{N} \frac{p_{i} e_{i}^{*}}{p_{i h}^{*}}\right)\right\} \\
& +2 T(1-T)\left\{\beta \beta^{*}\left(\sum_{i=1}^{N} \frac{p_{i}^{2}}{p_{i h}^{*}}\right)^{2}+\beta\left(\sum_{i=1}^{N} \frac{p_{i}^{3} e_{i}^{*}}{p_{i h}^{* 2}}+\sum_{j \neq i=1}^{N} \frac{p_{i}^{2} e_{j}^{*} p_{j}}{p_{i h}^{*} p_{j h}^{*}}\right)\right. \\
& \left.+\beta^{*}\left(\sum_{i=1}^{N} \frac{p_{i}^{3} e_{i}}{p_{i h}^{* 2}}+\sum_{j \neq i=1}^{N} \frac{p_{i}^{2} e_{j} p_{j}}{p_{i h}^{*} p_{j h}^{*}}\right)+\left(\sum_{i=1}^{N} \frac{e_{i} e_{i}^{*} p_{i}^{2}}{p_{i h}^{* 2}}+\sum_{j \neq i=1}^{N} \frac{e_{i} e_{j}^{*} p_{i} p_{j}}{p_{i h}^{*} p_{j h}^{*}}\right)\right\}
\end{aligned}
$$

Taking expected value, we have

$$
\begin{aligned}
E_{m}\left(B_{3}\right)= & T^{2}\left\{\beta^{2}\left(\sum_{i=1}^{N} \frac{p_{i}^{2}}{p_{i h}^{*}}\right)^{2}+a \sum_{i=1}^{N} \frac{p_{i}^{g+2}}{p_{i h}^{* 2}}\right\}+(1-T)^{2}\left\{\beta^{* 2}\left(\sum_{i=1}^{N} \frac{p_{i}^{2}}{p_{i h}^{*}}\right)^{2}+a \sum_{i=1}^{N} \frac{p_{i}^{g+2}}{p_{i h}^{* 2}}\right\} \\
& +2 T(1-T)\left\{\beta \beta^{*}\left(\sum_{i=1}^{N} \frac{p_{i}^{2}}{p_{i h}^{*}}\right)^{2}\right\} \\
= & a\left\{T^{2}+(1-T)^{2}\right\}\left(\sum_{i=1}^{N} \frac{p_{i}^{g+2}}{p_{i h}^{* 2}}\right)+T^{2} \beta^{2}\left(\sum_{i=1}^{N} \frac{p_{i}^{2}}{p_{i h}^{*}}\right)^{2}+(1-T)^{2} \beta^{* 2}\left(\sum_{i=1}^{N} \frac{p_{i}^{2}}{p_{i h}^{*}}\right)^{2} \\
& +2 T(1-T) \beta \beta^{*}\left(\sum_{i=1}^{N} \frac{p_{i}^{2}}{p_{i h}^{*}}\right)^{2} \\
= & a\left\{T^{2}+(1-T)^{2}\right\}\left(\sum_{i=1}^{N} \frac{p_{i}^{g+2}}{p_{i h}^{* 2}}\right)+\left[\left\{\beta T+(1-T) \beta^{*}\right\} \sum_{i=1}^{N} \frac{p_{i}^{2}}{p_{i h}^{*}}\right]^{2} .
\end{aligned}
$$


Now we have

$$
\begin{aligned}
E_{m}\left\{B\left(\widehat{Y}_{2}\right)_{h}\right\}^{2}= & E_{m}\left\{\sum_{i=1}^{N}\left(\frac{p_{i}}{p_{i h}^{*}}-1\right)\left(Y_{i}+\frac{1-T}{T} U_{i}\right)\right\}^{2} \\
= & E_{m}\left\{\sum_{i=1}^{N}\left(\frac{p_{i}}{p_{i h}^{*}}-1\right) Y_{i}+\frac{1-T}{T} \sum_{i=1}^{N}\left(\frac{p_{i}}{p_{i h}^{*}}-1\right) U_{i}\right\}^{2} \\
= & E_{m}\left\{\left\{\sum_{i=1}^{N}\left(\frac{p_{i}}{p_{i h}^{*}}-1\right) Y_{i}\right\}^{2}+\frac{(1-T)^{2}}{T^{2}}\left\{\sum_{i=1}^{N}\left(\frac{p_{i}}{p_{i h}^{*}}-1\right) U_{i}\right\}^{2}\right. \\
& \left.+\frac{2(1-T)}{T}\left\{\sum_{i=1}^{N}\left(\frac{p_{i}}{p_{i h}^{*}}-1\right)^{2} Y_{i} U_{i}+\sum_{j \neq i=1}^{N}\left(\frac{p_{i}}{p_{i h}^{*}}-1\right)\left(\frac{p_{j}}{p_{j h}^{*}}-1\right) Y_{i} U_{j}\right\}\right] \\
= & \beta^{2}\left(\sum_{i=1}^{N} \frac{p_{i}^{2}}{p_{i h}^{*}}-1\right)^{2}+a \sum_{i=1}^{N}\left(\frac{p_{i}^{g+2}}{p_{i h}^{* 2}}+p_{i}^{g}-2 \frac{p_{i}^{g+1}}{p_{i h}^{*}}\right) \\
& +\frac{(1-T)^{2}}{T^{2}}\left\{\beta^{* 2}\left(\sum_{i=1}^{N} \frac{p_{i}^{2}}{p_{i h}^{*}}-1\right)^{2}+a \sum_{i=1}^{N}\left(\frac{p_{i}^{g+2}}{p_{i h}^{* 2}}+p_{i}^{g}-2 \frac{p_{i}^{g+1}}{p_{i h}^{*}}\right)\right\} \\
& +2 \frac{1-T}{T}\left(\sum_{i=1}^{N} \frac{p_{i}^{2}}{p_{i h}^{*}}-1\right)^{2} \beta \beta^{*} \\
& +a\left\{1+\frac{(1-T)^{2}}{T^{2}}\right\} \sum_{i=1}^{N}\left(\frac{p_{i}^{g+2}}{p_{i h}^{* 2}}+p_{i}^{g}-2 \frac{p_{i}^{g+1}}{p_{i h}^{*}}\right) . \\
= & \left\{\beta\left(\sum_{i=1}^{N} \frac{p_{i}^{2}}{p_{i h}^{*}}-1\right)+\frac{1-T}{T} \beta^{*}\left(\sum_{i=1}^{N} \frac{p_{i}^{2}}{p_{i h}^{*}}-1\right)\right\}^{2} \\
& +a)^{2}
\end{aligned}
$$

On substituting $E\left(B_{1}\right), E\left(B_{2}\right), E\left(B_{3}\right)$ and $E_{m}\left(B\left(\widehat{Y}_{2}\right)_{h}\right)^{2}$ in $(A .1)$ and on re-arranging, we can get the following one:

$$
E_{m}\left[\operatorname{MSE}\left(\widehat{Y}_{2}\right)_{h}\right]=\frac{1}{n} A_{1}+A_{2}
$$

where

$$
\begin{aligned}
A_{1}= & {\left[\left(\frac{1-T}{T}\right)\left\{\left(\beta-\beta^{*}\right)^{2} \sum_{i=1}^{N} \frac{p_{i}^{3}}{p_{i h}^{* 2}}+2 a \sum_{i=1}^{N} \frac{p_{i}^{g+1}}{p_{i h}^{* 2}}\right\}\right.} \\
& +\frac{1}{T^{2}}\left\{a\left(T^{2}+(1-T)^{2}\right)\left(\sum_{i=1}^{N} \frac{p_{i}^{g+1}}{p_{i h}^{* 2}}-\sum_{i=1}^{N} \frac{p_{i}^{g+2}}{p_{i h}^{* 2}}\right)\right. \\
& \left.\left.+\left(\beta T+(1-T) \beta^{*}\right)^{2}\left(\sum_{i=1}^{N} \frac{p_{i}^{3}}{p_{i h}^{* 2}}-\left(\sum_{i=1}^{N} \frac{p_{i}^{2}}{p_{i h}^{*}}\right)^{2}\right)\right\}\right]
\end{aligned}
$$


and

$$
A_{2}=\left\{\left(\sum_{i=1}^{N} \frac{p_{i}^{2}}{p_{i h}^{*}}-1\right)\left(\beta+\frac{(1-T)}{T} \beta^{*}\right)\right\}^{2}+a\left\{1+\frac{(1-T)^{2}}{T^{2}}\right\} \sum_{i=1}^{N}\left(\frac{p_{i}^{g+2}}{p_{i h}^{* 2}}+p_{i}^{g}-2 \frac{p_{i}^{g+1}}{p_{i h}^{*}}\right)
$$

Proof: (Proof of Theorem 3.) Let $C_{1}$ be the cost per unit of collecting information of each individual. The cost $C_{0}$ of observing the sample of size $n$ is given by

$$
C_{0}=n C_{1} \text {. }
$$

To minimize $E_{m}\left[\operatorname{MSE}\left(\widehat{Y}_{2}\right)_{h}\right]$ subject to condition (A.2), consider the function

$$
L=\frac{1}{n} A_{1}+A_{2}+\lambda\left(n C_{1}-C_{0}\right) .
$$

Differentiating (A.3) partially with respect to $n$ and we get $n=C_{0} / C_{1}$ and hence (5.4) becomes (5.7), which proves the theorem.

\section{Appendix B:}

\begin{tabular}{|c|c|c|c|c|c|c|c|c|c|c|c|c|c|c|c|c|}
\hline \multirow{2}{*}{8} & \multirow[b]{2}{*}{$\rho$} & \multicolumn{5}{|c|}{$\overline{\rho^{*}=0.15}$} & \multicolumn{5}{|c|}{$\rho^{*}=0.65$} & \multicolumn{5}{|c|}{$\rho^{*}=0.95$} \\
\hline & & RE_1 & RE_2 & RE_3 & RE_4 & RE_5 & RE_1 & RE_2 & RE_3 & RE_4 & RE_5 & RE_1 & RE_2 & RE_3 & RE_4 & RE 5 \\
\hline & & \multicolumn{15}{|c|}{ Right Triangular } \\
\hline \multirow[t]{9}{*}{0} & .1 & 75.76 & 75.78 & 72.30 & 68.25 & 93.72 & 106.4 & 106.5 & 99.23 & 93.15 & 180.2 & 124.8 & 124.9 & 115.5 & 108.6 & 258.7 \\
\hline & .2 & 100.0 & 100.1 & 90.32 & 79.20 & 122.2 & 142.9 & 143.0 & 124.2 & 107.9 & 237.2 & 165.7 & 165.9 & 143.6 & 126.8 & 334.9 \\
\hline & .3 & 140.1 & 140.2 & 119.4 & 96.95 & 165.0 & 201.0 & 201.3 & 163.1 & 130.1 & 308.8 & 224.2 & 224.5 & 183.9 & 152.1 & 416.0 \\
\hline & .4 & 200.2 & 200.4 & 161.9 & 122.6 & 221.9 & 293.5 & 294.0 & 222.8 & 163.2 & 401.0 & 310.9 & 311.5 & 243.6 & 189.0 & 514.2 \\
\hline & .5 & 285.0 & 285.4 & 220.9 & 159.3 & 292.6 & 439.2 & 440.1 & 313.8 & 213.2 & 517.8 & 446.2 & 447.0 & 336.4 & 245.6 & 643.9 \\
\hline & .6 & 398.3 & $398: 8$ & 300.8 & 213.1 & 378.2 & 660.6 & 661.9 & 449.4 & 291.4 & 662.5 & 672.1 & 673.3 & 489.8 & 339.2 & 831.4 \\
\hline & .7 & 540.4 & 541.0 & 407.6 & 296.1 & 482.5 & 964.6 & 966.2 & 643.6 & 420.0 & 838.8 & 1089 & 1091 & 766.4 & 513.4 & 1135 \\
\hline & .8 & 706.6 & 707.2 & 553.0 & 434.7 & 617.0 & 1297 & 1298 & 904.3 & 645.1 & 1053 & 1977 & 1979 & 1338 & 905.4 & 1717 \\
\hline & .9 & 897.9 & 898.3 & 775.1 & 691.5 & 818.0 & 1515 & 1516 & 1231 & 1051 & 1312 & 3900 & 3904 & 2726 & 2083 & 3032 \\
\hline \multirow[t]{9}{*}{1} & .1 & 76.51 & 76.54 & 73.08 & 70.46 & 113.4 & 102.8 & 102.8 & 96.15 & 91.33 & 187.8 & 123.3 & 123.4 & 114.2 & 107.8 & 259.4 \\
\hline & .2 & 100.6 & 100.7 & 91.85 & 84.63 & 150.9 & 138.2 & 138.3 & 121.4 & 108.6 & 257.4 & 163.8 & 164.0 & 142.3 & 126.5 & 340.6 \\
\hline & .3 & 140.1 & 140.2 & 122.4 & 107.1 & 205.7 & 194.8 & 195.0 & 161.3 & 135.2 & 350.6 & 221.7 & 222.1 & 182.9 & 153.4 & 430.6 \\
\hline & .4 & 200.9 & 201.1 & 169.1 & 140.6 & 281.2 & 286.4 & 286.9 & 225.2 & 176.7 & 481.1 & 308.3 & 308.8 & 244.1 & 193.4 & 545.1 \\
\hline & .5 & 290.8 & 291.2 & 238.9 & 190.6 & 379.9 & 436.8 & 437.7 & 329.7 & 243.1 & 664.7 & 445.2 & 446.1 & 342.0 & 257.2 & 706.5 \\
\hline & .6 & 418.3 & 418.8 & 342.0 & 266.6 & 503.8 & 683.0 & 684.4 & 504.3 & 355.1 & 919.3 & 680.9 & 682.2 & 513.2 & 368.9 & 961.5 \\
\hline & .7 & 589.1 & 589.7 & 491.3 & 386.5 & 654.3 & 1065 & 1067 & 796.6 & 555.3 & 1255 & 1145 & 1147 & 857.0 & 595.6 & $14 \overline{33}$ \\
\hline & .8 & 799.4 & 799.9 & 698.9 & 580.5 & 832.3 & 1552 & 1553 & 1245 & 924.6 & 1634 & 2295 & 2298 & 1732 & 1189 & 2546 \\
\hline & .9 & 1029 & 1030 & 963.8 & 883.0 & 1035 & 1867 & 1868 & 1698 & 1485 & 1868 & 5883 & 5887 & 4752 & 3511 & 5856 \\
\hline \multirow[t]{9}{*}{2} & .1 & 77.93 & 77.97 & 74.07 & 71.38 & 128.3 & 101.9 & 102.0 & 95.21 & 90.50 & 197.1 & 122.8 & 122.9 & 113.7 & 107.3 & 261.9 \\
\hline & .2 & 103.2 & 103.3 & 93.63 & 86.43 & 173.7 & 137.9 & 138.0 & 120.9 & 108.4 & 276.9 & 163.3 & 163.5 & 141.9 & 126.3 & 346.8 \\
\hline & .3 & 144.1 & 144.2 & 125.2 & 110.1 & 238.2 & 195.5 & 195.8 & 161.7 & 136.2 & 385.9 & 221.6 & 222.0 & 182.7 & 153.5 & 442.4 \\
\hline & .4 & 207.5 & 207.8 & $\begin{array}{ll}174.2 \\
\end{array}$ & 145.9 & 326.9 & 290.0 & 290.5 & 227.8 & 179.8 & 543.1 & 309.3 & 309.8 & 244.8 & 194.4 & 566.1 \\
\hline & .5 & 303.0 & 303.4 & 248.8 & 200.0 & 442.8 & 448.3 & 449.2 & 338.3 & 251.0 & 772.5 & 449.2 & 450.0 & 345.0 & 260.0 & 743.8 \\
\hline & .6 & 440.8 & 441.4 & 361.6 & 283.7 & 586.4 & 716.3 & 717.8 & 529.2 & 373.8 & 1101 & 693.8 & 695.1 & 523.2 & 376.6 & 1033 \\
\hline & .7 & 627.9 & 628.6 & 528.0 & 417.0 & 755.0 & 1151 & 1153 & 864.2 & 600.1 & 1538 & 1190 & 1192 & 892.2 & 618.9 & 1590 \\
\hline & .8 & 855.9 & 856.5 & 758.6 & 631.3 & 939.5 & 1712 & 1723 & 1399 & 1029 & 1988 & 2497 & 2501 & 1895 & 1284 & 3018 \\
\hline & .9 & 1088 & 1088 & 1031 & 946.0 & 1119 & 2038 & 2039 & 1885 & 1645 & 2117 & 7194 & 7199 & 5890 & 4182 & 7886 \\
\hline
\end{tabular}

Relative efficiencies(RE $h$ ), $h=1,2,3,4,5$ of the proposed estimators for PPSWR sampling scheme using Unrelated Question Model under various distributions. 


\begin{tabular}{|c|c|c|c|c|c|c|c|c|c|c|c|c|c|c|c|c|}
\hline \multirow[b]{2}{*}{$g$} & \multirow[b]{2}{*}{$\rho$} & \multicolumn{5}{|c|}{$\rho^{*}=0.15$} & \multicolumn{5}{|c|}{$\rho^{*}=0.65$} & \multicolumn{5}{|c|}{$\rho^{*}=0.95$} \\
\hline & & RE I & RE 2 & RE 3 & RE 4 & RE 5 & RE_I & $\mathrm{RE}_{2}$ & RE 3 & RE_. & RE 5 & $\mathrm{RE} .1$ & $\mathrm{RE} 2$ & $\mathrm{RE}_{3} 3$ & RE_4 & RE 5 \\
\hline & & \multicolumn{15}{|c|}{ Exponential } \\
\hline 0 & .1 & 86.55 & 86.70 & 78.87 & 73.71 & 107.1 & 127.3 & 127.7 & 108.3 & 97.86 & 249.4 & 150.5 & 151.1 & 124.4 & 110.9 & 430.8 \\
\hline & .2 & 124.8 & 125.2 & 104.1 & 89.55 & 145.1 & 191.9 & 192.9 & 144.5 & 117.1 & 328.3 & 230.8 & 232.3 & 167.2 & 133.3 & 576.5 \\
\hline & .3 & 187.9 & $18 \overline{8.6}$ & 145.6 & 114.7 & 204.0 & 292.4 & 294.3 & 201.1 & 146.2 & 424.3 & 349.2 & 351.7 & 231.1 & 165.3 & 721.1 \\
\hline & .4 & 282.6 & 283.7 & 208.7 & 151.8 & 286.0 & 446.6 & 449.6 & 289.4 & 190.4 & 5478 & 526.6 & 530.6 & 329.3 & 213.0 & 888.2 \\
\hline & .5 & 416.4 & 418.1 & 302.7 & 207.6 & 396.4 & 675.2 & 679.4 & 428.3 & 260.2 & 711.5 & 797.0 & 802.8 & 487.1 & 288.8 & 1103 \\
\hline & .6 & 597.5 & 599.5 & 442.7 & 295.7 & 546.0 & 997.2 & 1002 & 648.8 & 376.9 & 936.6 & 1219 & 1226 & 755.5 & 420.1 & 1413 \\
\hline & .7 & 837.0 & 839.1 & 653.6 & $\overline{445.3}$ & 756.5 & 1426 & 1431 & 1001 & 589.0 & 1263 & 1910 & 1919 & 1254 & 679.4 & $\overline{1923}$ \\
\hline & .8 & 1163 & 1165 & 982.6 & 725.4 & 1073 & 1980 & 1983 & 1566 & 1019 & 1765 & 3195 & 3203 & 2325 & 1315 & 2951 \\
\hline & 9 & 1661 & 1663 & 1538 & 1316 & 1605 & 2699 & 2701 & 2447 & $197 !$ & 2560 & 6132 & 6138 & 5217 & 3562 & 5640 \\
\hline 1 & .1 & 94.12 & 94.36 & 83.41 & 77,36 & 165.8 & 124.5 & 125.0 & 105.6 & 95.6 & 290.5 & 148.8 & 149.4 & 123,0 & 109.8 & 443.6 \\
\hline & .2 & 136.7 & 137.3 & 111.0 & 94.78 & 223.4 & 190.2 & 191.2 & 142.9 & 116.3 & 406.7 & 228.9 & 230.3 & 165.9 & 1325 & 611.7 \\
\hline & .3 & 205.1 & 206.1 & 155.5 & 121.7 & 305.2 & 294.5 & 296.5 & 201.9 & 147.4 & 550.3 & 348.1 & 350.7 & 230.4 & 165.1 & 788.0 \\
\hline & .4 & 311.4 & 312.9 & $225 . \overline{4}$ & 162.3 & 417.9 & 462.1 & 465.3 & 296.9 & 195.1 & 739.3 & 530.6 & 534.7 & 331.1 & 214.1 & 1001 \\
\hline & .5 & $47 \overline{0.0}$ & 472.2 & 334.4 & 224.8 & 569.1 & 729.5 & 734.5 & 453.8 & 271.9 & 994.3 & 819.1 & 825.3 & 496.8 & 293.0 & $\overline{1288}$ \\
\hline & .6 & 696.4 & 699.2 & 504.1 & 325.9 & 769.9 & 1145 & 1152 & 720.1 & 404.0 & 1346 & 1297 & 1306 & 790.4 & 432.3 & 1719 \\
\hline & .7 & 1005 & 1008 & 768.4 & 501.3 & $\overline{1039}$ & 1754 & 1761 & 1180 & 653.5 & 1838 & 2158 & 2169 & 1373 & 716.9 & $2 \overline{4} 68$ \\
\hline & .8 & 1409 & 1412 & 1176 & 833.2 & 1408 & 2552 & 2558 & 1954 & 1182 & 2521 & 3989 & 4002 & 2775 & 1458 & 4086 \\
\hline & .9 & 1939 & 1940 & 1791 & 1503 & 1928 & 3358 & 3361 & 3020 & 2340 & 3312 & 8786 & 8796 & 7192 & 4415 & 8549 \\
\hline 2 & .1 & 102.6 & 103.0 & 87.78 & 80.25 & 239.7 & 125.3 & 125.8 & 104.8 & 94.38 & 345.4 & 148.0 & 148.6 & 122.0 & 108.9 & 462.0 \\
\hline & .2 & $\overline{152.9}$ & 153.7 & 118.4 & 98.95 & 333.7 & 195.2 & 196.4 & 143.6 & 116.0 & 511.5 & 229.1 & 230.6 & 165.3 & 131.8 & 653.5 \\
\hline & \begin{tabular}{|l|}
.3 \\
\end{tabular} & 231.1 & $2 \overline{32.5}$ & 167.0 & 127.4 & 456.0 & 307.3 & 309.6 & 205.4 & $1 \overline{48.4}$ & 724.8 & 351.1 & 353.8 & 230.7 & 164.9 & 862.8 \\
\hline & .4 & 354.0 & 356.1 & 244.5 & 170.9 & 620.0 & 493.3 & 497.3 & 307.0 & 198.7 & 1018 & 541.2 & 545.6 & 334.0 & 214.9 & 1126 \\
\hline & .5 & 543.4 & 546.6 & 369.1 & 239.1 & 834.6 & 807.5 & 814.1 & 481.4 & 280.8 & 1430 & 850.5 & 857.3 & 507.3 & 296.2 & 1495 \\
\hline & .6 & 824.5 & 828.7 & 570.7 & 351.9 & 1107 & 1337 & 1347 & 794.2 & 425.8 & 2009 & 1389 & 1399 & 824.3 & 441.9 & 2076 \\
\hline & .7 & 1214 & 1219 & 894.6 & $55 \overline{2.3}$ & 1443 & 2186 & 2199 & 1376 & 709.4 & 2795 & 2439 & 2453 & 1489 & 747.1 & 3158 \\
\hline & 8 & 1702 & 1706 & 1391 & 936.4 & 1840 & 3324 & 3335 & 2415 & 1337 & 3711 & 4996 & 5016 & 3267 & 1583 & 5760 \\
\hline & 9 & 1661 & 1663 & 1538 & 1316 & 1605 & 2699 & 2701 & 2447 & 1971 & 2560 & 6132 & 6138 & 5217 & 3562 & 5640 \\
\hline
\end{tabular}

\begin{tabular}{|c|c|c|c|c|c|c|c|c|c|c|c|c|c|c|c|c|}
\hline \multirow{3}{*}{$g$} & \multirow{3}{*}{$\rho$} & \multicolumn{5}{|c|}{$\rho^{*}=0.15$} & \multicolumn{5}{|c|}{$p^{*}=0.65$} & \multicolumn{5}{|c|}{$\rho^{*}=0.95$} \\
\hline & & RE_1 & $\mathrm{RE} 2$ & RE_3 & RE_4 & $\mathrm{RE} 55$ & RE_1 & RE_2 & $\mathrm{R} \overline{\mathrm{E}} 3 \mathrm{3}$ & RE_4 & $\mathrm{RE} 5$ & RE_1 & RE_2 & RE_3 & RE_4 & RE_5 \\
\hline & & \multicolumn{15}{|c|}{ Normal } \\
\hline \multirow[t]{9}{*}{0} & .1 & 80.39 & 80.47 & 75.32 & 71.17 & 98.84 & 114.7 & 115.0 & 103.0 & 95.27 & 206.0 & 135.2 & 135.5 & 119.2 & 109.5 & 321.4 \\
\hline & .2 & 109.7 & 109.9 & 95.95 & 84.60 & 129.6 & 161.9 & 162.4 & 132.5 & 112.3 & 269.2 & 191.3 & 192.0 & 153.6 & 129.7 & 421.2 \\
\hline & 3 & 158.1 & 158.5 & 129.7 & 106.3 & 176.6 & 236.0 & 237.0 & 178.4 & 138.3 & 346.8 & 271.9 & 273.1 & 203.6 & 158.2 & 523.3 \\
\hline & .4 & 230.7 & 231.4 & 180.2 & 138.4 & 240.8 & 351.6 & 353.2 & 249.6 & 177.9 & 446.3 & 391.3 & 393.1 & 278.7 & 200.5 & 643.9 \\
\hline & .5 & 332.9 & $33 \overline{3} .9$ & 253.2 & 185.7 & 324.7 & 527.8 & 530.2 & 360.2 & 239.7 & 576.2 & 574.7 & 577.4 & 397.0 & 266.8 & 801.5 \\
\hline & .6 & 469.2 & 470.5 & 357.9 & 257.9 & 434.0 & 783.6 & 786.8 & 532.1 & 340.8 & 749.6 & 870.3 & 874.1 & 595.7 & 380.3 & 1031 \\
\hline & .7 & 643.1 & 644.5 & 508.2 & 374.4 & 580.7 & 1124 & 1127 & 797.4 & 517.7 & 989.6 & 1384 & 1389 & 963.6 & 601.3 & 1413 \\
\hline & .8 & 862.4 & 863.5 & 728.8 & 576.9 & 789.5 & 1526 & 1529 & 1196 & 850.6 & 1334 & 2401 & 2407 & 1766 & 1133 & 2196 \\
\hline & .9 & 1162 & 1162 & 1073 & $957 . \overline{3}$ & $11 \overline{15}$ & 1934 & 1935 & $\overline{1741}$ & 1482 & 1813 & 4736 & 4741 & 3948 & 2929 & 4240 \\
\hline \multirow[t]{9}{*}{1} & .1 & 84.08 & 84.19 & 77.92 & 73.87 & 134.3 & 111.7 & 112.0 & 100.4 & 93.26 & 227.1 & 133.7 & 134.0 & 118.0 & 108.6 & 326.6 \\
\hline & .2 & 115.3 & 115.5 & 100.2 & 89.33 & 179.2 & 159.0 & 159.5 & 130.6 & 111.9 & 313.9 & 189.5 & 190.2 & 152.5 & 129.1 & 437.6 \\
\hline & .3 & 165.7 & 166.1 & 136.2 & 113.5 & 243.6 & 233.8 & 234.8 & 178.4 & 140.4 & 425.3 & 270.2 & 271.4 & 202.9 & 158.4 & 557.1 \\
\hline & .4 & 243.5 & 244.3 & 192.0 & 149.9 & 331,9 & 354.5 & 356.1 & 254.9 & 184.4 & 576.0 & 391.4 & 393.3 & 280.0 & 202,3 & 705.4 \\
\hline & .5 & 358.9 & 360.1 & 276.7 & 204.9 & 448.2 & 550.0 & 552.6 & 380.1 & 255.0 & 782.4 & 582.5 & 585.3 & 404.3 & 272.5 & 909.4 \\
\hline & .6 & 522.5 & 524.0 & 404.4 & 291.1 & 597.4 & 861.7 & 865.4 & 590.1 & 375.0 & 1066 & 905.3 & $909 . \overline{4}$ & 622.0 & 395.8 & 1224 \\
\hline & .7 & 741.1 & 742.7 & 594.5 & $43 \overline{2.9}$ & 787.3 & 1328 & 1332 & 944.6 & 595.1 & 1450 & 1515 & 1521 & 1055 & 646.4 & 1788 \\
\hline & 8 & 1014 & 1016 & 870 & 678.4 & 1029 & 1915 & 1919 & 1508 & 1027 & 1932 & 2917 & 2924 & 2126 & 1299 & 3068 \\
\hline & 9 & 1334 & 1335 & 1245 & 1104 & 1333 & 2374 & 2376 & 2158 & 1802 & 2355 & 6885 & 6893 & 5655 & 3865 & 6747 \\
\hline \multirow[t]{9}{*}{2} & .1 & 88.60 & 88.76 & 80.63 & 75.9 & 170.6 & 111.6 & 111.8 & 99.47 & 92.26 & 252.1 & 133,0 & 133.3 & 117.2 & 107.8 & 334.1 \\
\hline & .2 & 123.4 & 123.7 & 104.8 & 92.51 & 232.9 & 160.8 & 161.3 & 130.8 & 111.8 & 361.9 & 189.3 & 190.0 & 117.2 & 107.8 & 334.1 \\
\hline & .3 & 178.1 & 178.7 & 143.1 & 118.2 & 317.3 & $23 \overline{9} .1$ & 240.2 & $\overline{180.4}$ & 141.6 & 507.2 & 271.2 & 272.5 & 203.0 & 158.4 & 587.4 \\
\hline & .4 & 263.0 & 264.0 & 203.3 & $15 \overline{7.1}$ & 431.7 & 367.7 & 369.5 & 261.0 & 187.9 & 711.8 & 395.5 & 397.5 & 281.7 & 203.3 & 757.0 \\
\hline & .5 & 391.8 & 393.3 & 2969 & 217.0 & 580.9 & 583.5 & 586.6 & 397.0 & 263.6 & 1004 & 594.8 & 597.8 & 410.4 & 275.6 & 997.5 \\
\hline & .6 & 579.2 & 581.1 & 442.3 & 312.5 & 767.1 & 947.1 & 951.7 & 635.5 & 395.5 & 1417 & 1630 & 1637 & 1120 & 673.1 & 2115 \\
\hline & .7 & 833.8 & 835.8 & 663.5 & 472.3 & 989.3 & 1529 & 1534 & 1062 & 645.3 & 1972 & 1630 & 1637 & 1120 & 673.1 & 2115 \\
\hline & .8 & 1144 & 1145 & 980.3 & 749.2 & 1239 & 2284 & 2289 & 1771 & 1154 & 2570 & 3378 & 3388 & 2407 & 1408 & 3940 \\
\hline & 9 & 1462 & 1462 & 1368 & 1204 & 1493 & 2733 & 2735 & 2487 & 2036 & 2812 & 9293 & 9305 & 7402 & 4646 & 9918 \\
\hline
\end{tabular}




\begin{tabular}{|c|c|c|c|c|c|c|c|c|c|c|c|c|c|c|c|c|}
\hline \multirow{3}{*}{$g$} & \multirow[b]{2}{*}{$\rho$} & \multicolumn{5}{|c|}{$\overline{\rho^{\prime \prime}=0.15}$} & \multicolumn{5}{|c|}{$\rho^{*}=0.65$} & \multicolumn{5}{|c|}{$\rho^{*}=0.95$} \\
\hline & & RE_1 & RE_2 & RE_3 & RE_4 & RE_5 & RE_1 & RE_2 & RE_3 & RE_4 & RE.5 & RE_1 & RE_2 & RE_3 & RE_4 & RE_5 \\
\hline & & \multicolumn{15}{|c|}{ Chi Square } \\
\hline \multirow[t]{9}{*}{0} & .1 & 63.36 & 63.37 & 63.03 & 62.66 & 65.58 & 91.89 & 91.91 & 91.38 & 90.83 & 99.67 & 100.8 & 100.8 & 100.3 & 99.79 & 110.0 \\
\hline & .2 & 66.06 & 66.08 & 65.28 & 64.40 & 68.46 & $\overline{92.64}$ & 92.67 & 91.51 & 90.27 & 100.6 & 103.8 & 103.9 & 102.9 & 101.8 & 112.8 \\
\hline & .3 & 75.05 & 75.08 & 73.71 & 72.18 & 77.84 & 94.28 & 94.33 & 92.36 & 90.18 & 101.9 & 107.1 & 107.2 & 105.7 & 104.2 & 115.1 \\
\hline & .4. & 87.37 & 87.41 & 85.52 & 83.40 & 90.35 & 98.58 & 98.65 & 95.58 & 92.08 & 105.8 & 110.8 & 110.9 & 109.1 & 107.2 & 117.5 \\
\hline & .5 & 99.55 & 99.60 & 97.48 & 95.04 & 102.3 & 109.9 & 110.0 & 105.3 & 99.85 & 116.7 & 115.4 & 115.5 & 113.5 & 111.2 & 120.8 \\
\hline & 6 & 109.7 & 109.8 & 107.8 & 105.4 & 111.9 & 134.0 & 134.1 & 127.8 & 119.9 & 140.1 & 122.2 & 122.3 & 120.1 & 117.4 & 126.3 \\
\hline & .7 & 117.5 & 117.5 & 115.8 & 113.9 & 118.9 & 158.9 & 159.0 & 153.5 & 146.0 & 162.6 & 135.6 & 135.7 & 133.3 & 130.2 & 138.7 \\
\hline & .8 & 123.0 & 123.0 & 121.8 & 120.5 & 123.7 & 159.1 & 159.2 & 1567 & 153.2 & 160.5 & 182.5 & 182.6 & 179.4 & 174.8 & 185.3 \\
\hline & .9 & 126.5 & 126.5 & 125.9 & 125.2 & 126.7 & 145.9 & 145.9 & 145.1 & 144.2 & 146.2 & 642.3 & 642.4 & 629.7 & 604.7 & 651.2 \\
\hline \multirow[t]{9}{*}{1} & .1 & 63.68 & 63.69 & 63.38 & 63.05 & 68.05 & 91.50 & 91.71 & 91.20 & 90.67 & 100.3 & 100.8 & 100.8 & 100.3 & 99.76 & 110.1 \\
\hline & .2 & 66.84 & 66.86 & 66.11 & 65.33 & 71.78 & 92.66 & 92.69 & 91.55 & 90.35 & 101.9 & 103.8 & 103.9 & 102.9 & 101.8 & 113.0 \\
\hline & .3 & 76.08 & 76.11 & 74.82 & 73.42 & 81.48 & 94.65 & 94.70 & 92.79 & 90.70 & 104.0 & 107.1 & 107.2 & 105.8 & 104.3 & 115.3 \\
\hline & .4 & 88.54 & 88.59 & 86.81 & 84.82 & 93.85 & 99.56 & 99.64 & 96.68 & 93.3 & 109.3 & 110.9 & 111.0 & 109.2 & 107.3 & 117.8 \\
\hline & .5 & 100.8 & 100.9 & 98.84 & 96.51 & 105.3 & 111.9 & 112.1 & 107.6 & 102.2 & 122.4 & 115.6 & 115.7 & 113.7 & 111.4 & 121.3 \\
\hline & .6 & 110.9 & 111.0 & 109.1 & 106.8 & 114.3 & 137.8 & 137.9 & 131.8 & 124.0 & 148.7 & 122.5 & 122.6 & 120.4 & 117.8 & 127.0 \\
\hline & .7 & 118.5 & 118.6 & 117.0 & 115.0 & 120.6 & 163.4 & 163.5 & 158.3 & 150.8 & 170.7 & 136.3 & 136.4 & 134.1 & 131.0 & 139.9 \\
\hline & .8 & 123.7 & 123.8 & 122.6 & 121.3 & 124.7 & 161.8 & 161.9 & 159.5 & 156.1 & 164.3 & 184.6 & 184.7 & 181.7 & 177.1 & 188.5 \\
\hline & .9 & 126.9 & 126.9 & 126.3 & 125.6 & 127.2 & 146.8 & 146.8 & 146.0 & 145.1 & 147.3 & 676.0 & 676.2 & 663.9 & 637.5 & 639.7 \\
\hline \multirow[t]{9}{*}{2} & 1 & 64.00 & 64.00 & 63.70 & 63.40 & 70.13 & 91.56 & 91.58 & 91.07 & 90.56 & 100.9 & 100.8 & 100.8 & 100.2 & 99.73 & 110.2 \\
\hline & .2 & 67.52 & 67.54 & 66.82 & 66.08 & 74.59 & 92.70 & 92.73 & 91.61 & 90.45 & 102.9 & 103.8 & 103.9 & 102.9 & $10 t .8$ & 113.1 \\
\hline & .3 & 76.98 & 77.01 & 75.76 & 74.42 & 84.54 & 95.00 & 95.04 & 93.16 & 91.12 & 105.8 & 107.2 & 107.2 & 105.8 & 104.3 & 115.5 \\
\hline & .4 & 89.57 & 89.61 & 87.88 & 85.94 & 96.77 & 100.4 & 100.5 & 97.58 & 94.26 & 112.2 & 111.0 & 111.0 & 109.3 & 107.4 & 118.1 \\
\hline & .5 & 101.9 & 101.9 & 99.96 & 97.68 & 107.8 & 113.7 & 113.8 & 109.4 & 104.1 & 127.2 & 115.8 & 115.8 & 113.8 & 111.6 & 121.6 \\
\hline & .6 & 112.0 & 112.0 & 110.1 & 107.9 & 116.2 & 141.0 & 141.1 & 135.1 & 127.2 & 155.8 & 122.8 & 122.9 & 120.7 & 118.1 & 127.6 \\
\hline & .7 & 119.4 & 119.4 & 117.8 & 116.0 & 121.9 & 167.1 & 167.2 & 162.1 & 154.6 & $\begin{array}{l}177.3 \\
\end{array}$ & 136.9 & 136.9 & 134.6 & 131.6 & 140.9 \\
\hline & .8 & 124.3 & 124.4 & 123.2 & 121.9 & 125.5 & 164.0 & 164.1 & 161.8 & 158.4 & 167.4 & 186.4 & 186.4 & 183.5 & 178.9 & 190.9 \\
\hline & .9 & 127.2 & 127.2 & 126.6 & 126.0 & 127.5 & 147.5 & 147.5 & 146.8 & 145.8 & 148.1 & 703.7 & 703.8 & 691.7 & 663.9 & 728.7 \\
\hline
\end{tabular}

\begin{tabular}{|c|c|c|c|c|c|c|c|c|c|c|c|c|c|c|c|c|}
\hline \multirow[b]{2}{*}{$g$} & \multirow[b]{2}{*}{$\rho$} & \multicolumn{5}{|c|}{$\rho^{*}=0.15$} & \multicolumn{5}{|c|}{$\rho^{*}=0.65$} & \multicolumn{5}{|c|}{$\rho^{*}=0.95$} \\
\hline & & RE_I & RE_2 & $\overline{R E 3}$ & RE_4 & RE_5 & RE_1 & RE_2 & RE_3 & $\overline{\text { RE_4 }}$ & RE_5 & RE_1 & RE_2 & RE_3 & RE_4 & RE_5 \\
\hline & & \multicolumn{15}{|c|}{ Gamma } \\
\hline \multirow[t]{9}{*}{0} & .1 & 82.76 & 82.93 & 77.84 & 73.73 & 99.20 & 13.9 & 114.4 & 102.9 & 95.46 & 202.4 & 133.6 & 134.2 & 118.5 & 109.1 & 307.0 \\
\hline & .2 & 110.4 & 110.8 & 97.49 & 86.24 & 134.2 & 158.4 & 159.4 & 131.3 & 111.9 & 263.4 & 186.6 & 188.0 & 151.6 & 128.7 & 398.2 \\
\hline & .3 & 156.6 & 156.4 & 129.6 & 106.6 & 179.5 & 227.6 & 229.4 & 175.4 & 136.9 & 338.8 & 261.5 & 263.8 & 199.3 & 156.3 & 492.4 \\
\hline & .4 & 223.5 & 224.8 & 177.7 & 136.7 & 241.1 & 335.2 & 338.2 & 243.6 & 174.8 & 435.9 & 371.1 & 374.6 & 270.5 & 197.0 & 604.5 \\
\hline & .5 & 319.3 & 321.2 & 246.9 & 181.0 & 320.7 & 500.0 & 504.5 & 349.2 & 233.6 & 561.8 & 538.2 & 543.2 & 381.9 & 260.4 & 751.8 \\
\hline & .6 & 447.7 & 450.1 & 344.7 & 248.1 & 422.1 & 742.5 & 748.4 & 511.7 & 329.0 & 726.4 & 808.5 & 815.3 & 567.7 & 368.2 & 966.1 \\
\hline & .7 & 611.2 & 613.7 & 482.2 & 355.2 & 554.3 & 1070 & 1076 & 757.9 & 493.2 & 946.4 & 1286 & 1295 & 909.4 & 576.2 & 1322 \\
\hline & .8 & 812.3 & 814.5 & 678.8 & 538.0 & 737.2 & 1449 & 1455 & 1116 & 795.8 & 1249 & 2257 & 2268 & 1647 & 1071 & 2043 \\
\hline & .9 & 1071 & 1073 & 979.2 & 875.4 & 1018 & 1790 & 1792 & 1583 & 1352 & 1650 & 4466 & 4477 & 3608 & 2700 & 3876 \\
\hline \multirow[t]{9}{*}{1} & .1 & 88.82 & 88.47 & 81.60 & 77.30 & 99.70 & 112.4 & 112.9 & 10 & 94.2 & 226.2 & 132.5 & 133.1 & 117.5 & 108.3 & 313.1 \\
\hline & .2 & 119.0 & 119.5 & 103.6 & 92.41 & 186.6 & 158.1 & 159.1 & 130.8 & 112.6 & 308.4 & 185.6 & 186.9 & 150.8 & 128.4 & 413.9 \\
\hline & .3 & 167.2 & 168.1 & 138.5 & 115.9 & 247.7 & $22 \overline{9.2}$ & 231.1 & 177.2 & 140.5 & 414.0 & 261.1 & 263.4 & 199.2 & 156.9 & 522.5 \\
\hline & .4 & 240.4 & 241.9 & 192.1 & 151.2 & 331.0 & 342.4 & 345.5 & 251.1 & 183.6 & 557.7 & 372.7 & 376.3 & 272.5 & 199.6 & 658.0 \\
\hline & .5 & 347.7 & 349.9 & 273.0 & 204.4 & 440.2 & 524.3 & 529.2 & 371.8 & 252.8 & 755.9 & 546.7 & 551.9 & 390.1 & 267.6 & 846.0 \\
\hline & .6 & 498.3 & 501.1 & 393.7 & 287.3 & 579.2 & 813.6 & 820.6 & 573.2 & 370.3 & 1029 & 839.3 & 846.5 & 595.0 & 386.8 & 1138 \\
\hline & .7 & 698.5 & 701.4 & 570.7 & 422.0 & 753.0 & 1248 & 1256 & 910.3 & 584.0 & 1394 & 1396 & 1406 & 1002 & 629.0 & 1669 \\
\hline & .8 & 946.2 & 948.6 & 821.3 & 649.9 & 968.4 & 1795 & 1802 & 1435 & 995.6 & 1837 & 2704 & 2718 & 2015 & 1261 & 2888 \\
\hline & .9 & 1229 & 1230 & 1151 & 1030 & 1230 & 2194 & 2197 & 2003 & 1693 & 2183 & 6530 & 6545 & 5387 & 3742 & 6436 \\
\hline \multirow[t]{9}{*}{2} & .1 & 94.97 & 95.34 & 85.91 & 80.53 & 99.80 & 113.8 & 114.3 & 101.4 & 93.96 & 258.3 & 132.2 & 132.8 & \begin{tabular}{|l|l}
117.0 \\
\end{tabular} & 107.8 & 322.7 \\
\hline & .2 & 131.0 & 131.8 & 110.6 & 97.17 & 252.2 & 162.6 & 163.8 & 132.5 & 113.4 & 364.6 & 186.2 & 187.6 & 150.7 & 128.1 & 432.6 \\
\hline & .3 & 185.3 & 186.6 & 148.7 & 122.4 & 332.7 & 238.8 & 241.0 & 181.3 & 142.8 & 502.8 & 263.5 & 265.9 & 200.0 & 157.2 & 553.4 \\
\hline & .4 & 267.2 & 269.2 & 207.4 & 160.4 & 439.0 & 361.8 & 365.5 & 260.1 & 188.5 & 696.0 & 378.9 & 382.6 & 275.2 & 200.9 & 707.3 \\
\hline & .5 & 388.3 & 391.3 & 297.6 & 218.7 & 575.2 & 565.4 & 571.4 & 392.0 & 263.0 & 971.0 & 561.5 & 567.0 & 397.4 & 271.2 & 926.1 \\
\hline & .6 & 560.7 & 564.4 & 435.1 & 310.9 & 742.5 & 904.2 & 913.1 & 621.3 & 392.4 & 1358 & 876.2 & 884.3 & 615.1 & 396.2 & 1278 \\
\hline & .7 & 790.0 & 793.9 & 639.8 & 462.7 & 938.8 & 1440 & 1451 & 1026 & 635.5 & 1869 & 1501 & 1513 & 1065 & 656.0 & 1950 \\
\hline & .8 & 1063 & 1066 & 924.2 & 718.7 & 1155 & 2122 & 2132 & 1680 & 1119 & 2402 & 3102 & 3120 & 2273 & 1367 & 3642 \\
\hline & .9 & 1337 & 1338 & 1259 & 1120 & 1368 & 2496 & 2500 & 2291 & 1906 & 2575 & 8617 & 8640 & 6980 & 4492 & 9258 \\
\hline
\end{tabular}




\section{References}

Amahia, G. N., Chaubey, Y. P. and Rao, T. J. (1989). Efficiency of a new estimator in PPS sampling for multiple characteristics, Journal of Statistical Planning and Inference, 21, 72-84.

Arnab, R. (2001). Estimation of a finite population total in varying probability sampling for multicharacter surveys, Metrika, 54, 159-177.

Bansal, M. L. and Singh, R. (1985). An alternative estimator for multiple characteristics in PPS sampling, Journal of Statistical Planning and Inference, 11, 313-20.

Bansal, M. L., Singh, S. and Singh, R. (1994). Multi-character survey using randomized response technique, Communications in Statistics - Theory and Methods, 23, 1705-1715.

Greenberg, B. G., Kuebler, R. R., Abernathy, J. R. and Horvitz, D. G. (1971). Application of the randomized response technique in obtaining quantitative data, Journal of the American Statistical Association, 66, 243-250.

Grewal, I. S., Bansal, M. L. and Singh, S. (1997). An alternative estimator for multiple characteristics using randomized response technique in pps sampling, The Aligarh Journal of Statistics, 19, $51-65$.

Hansen, M. H. and Hurwitz, W. N. (1943). On the theory of sampling from finite populations, The Annals of Mathematical Statistics, 14, 333-362.

Rao, J. N. K. (1966). Alternative estimators in PPS sampling for multiple characteristics, Sankhyā: The Indian Journal of Statistics, Series A, 28, 47-60.

Singh, S. (2003). Advanced Sampling Theory with Applications: How Michael "Selected" Amy, Kluwer Academic Publishers, Boston.

Warner, S. L. (1965). Randomized response: A survey technique for eliminating evasive answer bias, Journal of the American Statistical Association, 60, 63-69. 